\title{
Prevalencia de Síndrome de Burnout en un Centro de Cirugía Académico-Asistencial Público en Chile
}

\author{
Paula Astudillo M. ${ }^{1,2,3}$, Héctor Losada M. ${ }^{1,4}$, \\ Pilar Schneeberger H. ${ }^{1,4}$, Felipe Coronado ${ }^{1,4}$ y Sonia Curitol S. ${ }^{1}$,
}

\section{Prevalence of Burnout Syndrome in a Center for Academic-Public Surgery Assistance in Chile}

\begin{abstract}
Aim: To assess the degree of emotional exhaustion, personal accomplishment and depersonalization in the work of physicians of the Hospital Hernán Henríquez Aravena surgery service and department of surgery of the Universidad de La Frontera, Temuco, Chile. Material and Method: Cross-sectional study. 19 medical interns, 11 surgical residents and 15 surgeons of Hernán Henríquez Aravena Hospital and surgery department of Universidad de La Frontera were included. Applied instrument: MBI questionnaire in its adaptation of the Spanish population. Calculation of measures of central tendency and percentage, as well as comparison of variables through t Student and values of Cronbach's alpha coefficients were performed. Results: Regarding the MBI scale, the overall prevalence of the syndrome was $64.4 \%$, whereas, by dimensions, emotional exhaustion showed a prevalence of $76 \%$; Low personal accomplishment at work, $55 \%$, and depersonalization $62 \%$. Statistically significant differences were found in emotional exhaustion and depersonalization according to socio-demographic variables: men and women, single and married, subjects without children and with children, whether or not they take emergency room shifts, obtaining higher levels in women, unmarried, subjects without children and those who take emergency shifts. Conclusions: It is accepted that the central triggering factor is the high emotional exhaustion that gradually leads to a state of emotional and cognitive distancing in their daily activities, with the consequent inability to respond to demands of the service. In this distancing occurs depersonalization, indifference and cynical attitudes toward responsibilities or towards people. Therefore, measures should be taken to try to reduce the prevalence of this syndrome in our medical interns and residents, mainly.
\end{abstract}

Key words: burnout, professional; stress, psychological; medical staff, hospital; surgery department, hospital.

\section{Resumen}

Objetivo: Evaluar el grado de Síndrome de Burnout en el quehacer de los médicos del Servicio de Cirugía Hospital Hernán Henríquez Aravena y Departamento de Cirugía de la Universidad de La Frontera, Temuco, Chile, valorando los niveles de agotamiento emocional, realización personal y despersonalización. Material y Método: Estudio de corte transversal. Se incluyeron 19 internos de medicina, 11 residentes de cirugía y 15 cirujanos pertenecientes al Servicio de Cirugía del Hospital Hernán Henríquez Aravena y Departamento de Cirugía de la Universidad de La Frontera. Instrumento aplicado: cuestionario MBI. Se realizó cálculo de medidas de tendencia central y porcentaje, así como comparación de variables a través de $t$ de Student y valores de coeficientes de alfa de Cronbach. Resultados: Respecto a la escala de MBI, la prevalencia global del síndrome fue del $64,4 \%$, mientras que, por dimensiones, el cansancio emocional mostró una prevalencia del 76\%; la baja realización personal en el trabajo, el 55\%, y la despersonalización el 62\%. Se obtuvo diferencias estadísticamente significativas en cansancio emocional y despersonalización según variables sociodemográficas: hombres y mujeres, solteros y casados, sujetos sin hijos y con hijos, si hace o no turnos de urgencia, obteniendo niveles más altos en las mujeres, en solteros, en sujetos sin hijos y los que hacen turnos de urgencia. Conclusión: Se acepta que el factor central desencadenante es el excesivo agotamiento emocional que
'Departamento de

Cirugía, Traumatología y

Anestesióloga. Universidad de

La Frontera, Temuco, Chile.

2Programa Doctorado en

Psicología, Salud y Calidad de

vida. Universidad de Girona,

Girona, España.

3Programa de Doctorado y

Magíster en Ciencias Médicas,

Universidad de La Frontera,

Temuco, Chile.

${ }^{4}$ Programa de Especialidad

en Cirugía, Universidad de La

Frontera, Temuco, Chile

Financiado por la vicerrectoría de investigación y postgrado. Universidad de La Frontera. Temuco. Chile.

Recibido el 21 de abril de 2017 y aceptado para publicación el 30 de agosto de 2017.

Correspondencia a: Dr. Héctor Losada M. hector.losada@ufrontera.cl 


\section{ARTíCULO ORIGINAL}

gradualmente lleva a un estado de distanciamiento emocional y cognitivo en sus actividades diarias, con la consecuente incapacidad de responder a las demandas del servicio. En este distanciamiento ocurre una despersonalización, indiferencia, y actitudes vinculadas al sarcasmo y la ironía hacia las responsabilidades o hacia las personas, una tendencia de no creer en la sinceridad del ser humano. Por tanto se deben tomar medidas para intentar reducir la prevalencia de este síndrome, principalmente en nuestros internos y residentes.

Palabras clave: síndrome de burnout, estrés psicológico, personal médico, departamento de cirugía.

\section{Introducción}

En general la prevalencia del estrés crónico en los profesionales médicos es alta ${ }^{1-3}$, documentándose en residentes de especialidades ${ }^{4-6}$ y alcanzando un $35 \%$ en residentes en cirugía ${ }^{7}$.

El síndrome de Burnout o de "estar quemado" con el trabajo 8,9 es el cansancio emocional que lleva a una pérdida de motivación y que suele progresar hacia sentimientos de inadecuación y fracaso. Se compone de tres dimensiones: a) agotamiento o cansancio emocional definido como el cansancio y fatiga que puede manifestarse física y/o psíquicamente, es la sensación descrita como no poder dar más de sí mismo a los demás; b) despersonalización como desarrollo de sentimientos, actitudes y respuestas negativas, distantes y frías hacia otras personas especialmente hacia los pacientes; c) la baja realización personal o logro que se caracteriza por una dolorosa desilusión para dar sentido a la propia vida y hacia los logros personales consentimientos de fracaso y baja autoestima ${ }^{7,10-20}$. Por otra parte, las consecuencias del estrés laboral no afectan sólo a las personas que lo viven. Los efectos pueden incidir también sobre la organización. De este modo, posibles consecuencias del estrés en el trabajo son las pérdidas en el trabajo, el incremento del ausentismo, las ineficiencias, los errores, entre otras ${ }^{21}$. Al aproximarnos al contexto sociopolítico en el cual fluyen los procesos, la IX Región es la que presenta históricamente los indicadores biomédicos y sociales más deteriorados del país con los índices de pobreza e indigencia más altos ${ }^{22}$. El trabajo que se desarrolla en un servicio quirúrgico en este contexto requiere notable experiencia clínica y madurez profesional que permita hacer frente a la frecuente toma de decisiones difíciles con implicaciones éticas-morales y el enfrentamiento diario al sufrimiento humano, pacientes terminales y muerte. Así, la vivencia de un estrés intenso y/o crónico puede tener consecuencias negativas sobre la persona. Esto puede reflejarse en problemas cardiovasculares, musculoesqueléticos, salud mental como suicidio, crisis nerviosas o en patologías gastrointestinales, heridas en el lugar de trabajo, funciones inmunes afectadas, entre otras ${ }^{23,24}$.

Frente a este panorama surge la necesidad de evaluar la prevalencia global del Síndrome de Burnout en el quehacer de los médicos del Servicio de Cirugía Hospital Hernán Henríquez Aravena y Departamento de Cirugía de la Universidad de La Frontera, Temuco, Chile, año 2016, valorando los niveles de agotamiento emocional, realización personal y despersonalización en la dinámica de relaciones médicas asistenciales y académicas; de académicos, residentes e internos de medicina.

Por otro lado, la exploración pretende ser un primer paso al conocimiento de los esfuerzos cooperativos organizados en torno a enfrentar el estrés en instituciones de salud de alta complejidad, útil no sólo para los planificadores y trabajadores en salud, sino también para orientar de manera objetiva las futuras proposiciones de promoción de salud de los trabajadores, lo que impactará directamente a los usuarios.

\section{Material y método}

\section{Diseño}

Estudio de corte transversal.

\section{Sujetos}

Cuarenta y cinco participantes: internos de medicina, residentes de cirugía, cirujanos del departamento de cirugía de la Universidad de La Frontera y del Servicio de Cirugía Adulto del HHHA.

\section{Instrumento}

Se aplicó cuestionario MBI (Maslach y Jackson, $1981)^{25,26}$ en su adaptación a la población española (Seisdedos, 1997) ${ }^{27}$.

Es un instrumento en el que se plantea al sujeto una serie de enunciados sobre los sentimientos y pensamientos con relación a su interacción con el trabajo.

Está formado por 22 ítems que se valoran con una escala tipo Likert. El sujeto valora, mediante un 
rango de 6 adjetivos que van de "nunca" a "diariamente", con qué frecuencia experimenta cada una de las situaciones descritas en los ítems. Consta de tres subescalas 1) agotamiento emocional, 2) despersonalización y 3) realización personal en el trabajo.

La subescala de Agotamiento Emocional o cansancio emocional (CE) está formada por 9 ítems que refieren la disminución o pérdida de recursos emocionales o describen sentimientos de estar saturado y cansado emocionalmente por el trabajo; la subescala de Despersonalización (DE) está formada por 5 ítems que describen una respuesta fría e impersonal y falta de sentimientos e insensibilidad hacia los sujetos objeto de atención; y la subescala de Realización Personal en el trabajo (RP) está compuesta por 8 ítems que describen sentimientos de competencia y eficacia en el trabajo. Tendencia a evaluar el propio trabajo de forma negativa y vivencia de insuficiencia profesional.

\section{Maniobra}

La segunda quincena del mes de julio del año 2016, se congregó a las personas en sala de reunión clínica donde se les entregó, previo consentimiento, el cuestionario junto a una breve encuesta en donde informaron datos sociodemográficos como edad, estado civil, número de hijos, horas de sueño, si realiza turnos de urgencia. Se proporcionó instrucción necesaria para su llenado de cuestionario MBI y se pidió expresamente que a cada una de las frases debiera responder expresando la frecuencia con que tenía ese sentimiento, poniendo una cruz en la casilla correspondiente y número que considerará más adecuado. No tomó más de 15 min en total.

\section{Herramientas estadísticas}

Tanto el "constructo de quemarse" como cada una de sus dimensiones fueron consideradas como variables continuas. Se construye base de datos en programa Acces ${ }^{\circledR}$, luego se trabaja en programa Excel $^{\circledR}$, Stata9. $0^{\circledR}$. Se realizaron cálculos de medidas de tendencia central y porcentaje, así como comparación de variables a través de $t$ student. Para evaluar los valores de los coeficientes de alfa de Cronbach, se trabajó bajo las recomendaciones de George y
Mallery ${ }^{28}$ siendo: mayor a 0,7 aceptable, mayor a 0,6 cuestionable y mayor a 0,5 pobre.

Las puntuaciones de "Burnout" se calificaron según la norma americana y la adaptación catalana, que determinan las categorías de bajo, medio y alto en cada una de las dimensiones. Se considerará con "Burnout" cuando cualquiera de las tres dimensiones se encuentra en las categorías media y alta $^{29}$. La prevalencia de "Burnout" es el porcentaje de sujetos con puntaje medio y alto según el total de sujetos del grupo (Tabla 1).

\section{Consideraciones éticas}

La participación fue completamente voluntaria, pudiendo elegir no participar en ella y no contestar cualquier pregunta que considerara inoportuna o incomoda y detenerse en cualquier momento. No se identificó el nombre. Se realizaron todos los esfuerzos para mantener la confidencialidad y anonimato de la participación. Sin embargo, se les comunicó que la información provista será combinada con otras del mismo estudio para publicaciones o presentaciones en congresos. Se respondieron todas sus preguntas y se aclararon sus dudas. No hubo riesgos identificables asociados con este estudio. El estudio fue aprobado por el grupo de investigación del departamento de cirugía, traumatología y anestesióloga de la Universidad de La Frontera y por el Servicio de Cirugía del HHHA.

\section{Financiamiento}

El proyecto fue financiado por la vicerrectoría de investigación y postgrado y el Departamento de Cirugía de la Universidad de La Frontera.

\section{Resultados}

Se buscaron valores fuera de rangos (outliers o valor atípico), numéricamente distante del resto de los datos, para ello se calculó rango, desviación estándar, promedio individual, coeficiente de variación, Mahalanobis. No existió caso para clasificarlo como atípico quedando un número de 45 sujetos analizados.

Tabla 1. Puntuaciones y niveles de identificación de dimensiones de Burnout

\begin{tabular}{|cccc|}
\hline Agotamiento emocional & Baja realización en el trabajo & Despersonalización & Nivel de identificación \\
$>$ o igual a 27 & $<$ o igual a 30 & $>$ o igual a 14 & Alto \\
$17-26$ & $36-31$ & $9-13$ & Medio \\
$0-16$ & $48-37$ & $0-8$ & Bajo \\
\hline
\end{tabular}




\section{ARTíCULO ORIGINAL}

Tabla 2. Número de internos de medicina y porcentaje según niveles de puntuación (n: 19)

\begin{tabular}{|lccc|}
\hline & Alto & Medio & Bajo \\
Cansancio emocional & $17(89 \%)$ & $2(11 \%)$ & $0(0 \%)$ \\
Realización personal & $\begin{array}{c}3(16 \%) \\
\text { baja realización } \\
\text { personal }\end{array}$ & $9(47 \%)$ & $\begin{array}{c}7(37 \%) \\
\text { sensación de } \\
\text { logro }\end{array}$ \\
Despersonalización & $11(58 \%)$ & $3(16 \%)$ & $5(26 \%)$ \\
Total & 31 & 14 & 12 \\
\hline
\end{tabular}

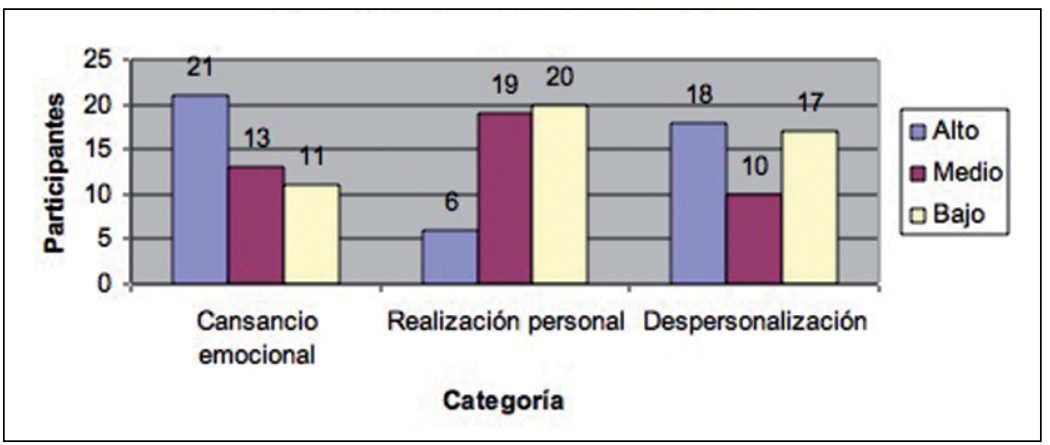

Figura 1. Número total de participantes según categoría en cada subescala MBI.

Tabla 3. Número de residentes y porcentaje según niveles de puntuación (n: 10)

\begin{tabular}{|llcc|}
\hline & Alto & Medio & Bajo \\
Cansancio emocional & $3(30 \%)$ & $5(50 \%)$ & $2(20 \%)$ \\
Realización personal & $2(20 \%)$ & $2(20 \%)$ & $6(60 \%)$ \\
Despersonalización & $4(40 \%)$ & $3(30 \%)$ & $3(30 \%)$ \\
Total & 9 & 10 & 11 \\
\hline
\end{tabular}

Tabla 4. Número de médicos especialistas académicos y asistenciales y porcentaje según niveles de puntuación (n: 16)

\begin{tabular}{|lccc|}
\hline & Alto & Medio & Bajo \\
Cansancio emocional & $1(6 \%)$ & $6(38 \%)$ & $9(56 \%)$ \\
Realización personal & $1(6 \%)$ & $8(50 \%)$ & $7(44 \%)$ \\
Despersonalización & $3(19 \%)$ & $4(25 \%)$ & $9(56 \%)$ \\
Total & 5 & 18 & 25 \\
\hline
\end{tabular}

Tabla 5. Número de hombres y porcentaje según niveles de puntuación (n: 31)

\begin{tabular}{|lccc|}
\hline & Alto & Medio & Bajo \\
Cansancio emocional & $11(35 \%)$ & $9(29 \%)$ & $11(36 \%)$ \\
Realización personal & $3(10 \%)$ & $13(42 \%)$ & $15(48 \%)$ \\
Despersonalización & $12(39 \%)$ & $8(26 \%)$ & $11(35 \%)$ \\
Total & 26 & 30 & 37 \\
\hline
\end{tabular}

El 31\% (14) es femenino, 69\% (31) masculino, con un promedio de edad de $33 \pm 13,7$ años con un mínimo de 22 y un máximo de 75.

Respecto al estado civil 27\% (12) son casados, 64\% (29) son solteros. El 69\% (31) no tiene hijos. El $100 \%$ reside en zona urbana.

De la muestra 43\% (19) corresponde a internos de medicina, $23 \%$ (11) son residentes $(50 \%$ son de primer año), 34\% (15) académicos-asistenciales.

Sólo aplicado a residentes y académicos asistenciales (26), el 54\% (14) declara su desempeño laboral sólo en el área pública y el 46\% (12) en área pública y privada.

Acerca de la modalidad de turnos el 73\% (33) realiza turnos de urgencia (Tabla 12).

De un total de 43 sujetos (2 no reportan el dato), un $81 \%$ (35) declara dormir menos de $7 \mathrm{~h}$ y un $19 \%$ (8) más de 7 h (Tabla 13 , Tabla 14).

Sólo aplicado a residentes y académicos asistenciales (26), la experiencia en el lugar de trabajo tuvo un promedio de $10 \pm 12,8$ años con un mínimo de 0 a 47 años.

Ante la pregunta: ¿siente estrés hoy?, con una respuesta numérica de 1 a 10 siendo 10 la máxima expresión de estrés, declaran un promedio de $6 \pm$ 2,9, con un mínimo de 0 y un máximo de 10 .

La consistencia interna de la escala de MBI total tiene un valor alpha de Cronbach de 0,82 . Por cada subescala Agotamiento o Cansancio emocional (CE) un valor de 0,92, Realización Personal (RP) 0,73 y Despersonalización (DE) un alpha de 0,79.

Respecto a la escala de MBI, la prevalencia global del síndrome fue del $64,4 \%$, mientras que, por dimensiones, el cansancio emocional mostró una prevalencia del 76\% (34 sujetos o participantes); la baja realización personal en el trabajo, el 55\% (25), y la despersonalización el 62\% (28) (Figura 1).

En los internos de medicina la prevalencia fue del $79 \%$, mientras que el CE mostró una prevalencia del 100\% (19); la baja RP, el 63\% (12), y DE, el 74\% (14) (Tabla 2). En los residentes la prevalencia fue del $63 \%$, mientras que el CE mostró una prevalencia del 80\% (8); la baja RP, el 40\% (4), y DE, el 70\% (7) (Tabla 3). La prevalencia en los médicos especialistas académicos y asistenciales fue del $48 \%$, mientras que el CE mostró una prevalencia del $44 \%$ (7 sujetos); la baja RP, el 56\% (9), y DE el 44\% (7) (Tabla 4).

Al explorar las variables sociodemográficas encontramos que la prevalencia del síndrome en hombres fue del $60 \%$, mientras que CE mostró una prevalencia del 64\% (20); la baja RP, el 52\% (16), y DE, el 65\% (20) (Tabla 5). La prevalencia en mujeres fue del 74\%, mientras que el CE mostró una 
prevalencia del 100\% (14); la baja RP, el 64\% (9), y la DE, el $57 \%$ (8) (Tabla 6). La prevalencia en los sujetos sin hijos fue del $72 \%$, mientras que el CE mostró una prevalencia del $87 \%$ (27); la baja RP, el 58\% (18), y DE, el 71\% (22) (Tabla 7). En los sujetos con hijos la prevalencia del síndrome fue de un $48 \%$, mientras que el CE mostró una prevalencia del 50\% (7); la baja RP, el 50\% (7), y DE el 43\% (6) (Tabla 8). En los sujetos solteros la prevalencia del síndrome fue de un $71 \%$, mientras que el CE mostró una prevalencia del $86 \%$ (25); la baja RP, el 59\% (17), y DE, el 69\% (20) (Tabla 9). En los sujetos casados la prevalencia del síndrome fue de un $44 \%$, mientras que el CE mostró una prevalencia del 29\% (6); la baja RP, el $42 \%$ (5), y DE el75\% (5) (Tabla 10).

Respecto a los que no hacen turno de urgencia se observa

Tabla 6. Número de mujeres y porcentaje según niveles de puntuación (n: 14)

\begin{tabular}{|lccc|}
\hline & Alto & Medio & Bajo \\
Cansancio emocional & $10(71 \%)$ & $4(29 \%)$ & $0(0 \%)$ \\
Realización personal & $3(21 \%)$ & $6(43 \%)$ & $5(36 \%)$ \\
Despersonalización & $6(43 \%)$ & $2(14 \%)$ & $6(43 \%)$ \\
Total & 19 & 12 & 11 \\
\hline
\end{tabular}

Tabla 8. Número de sujetos con hijos y porcentaje según niveles de puntuación (n: 14)

\begin{tabular}{|llll|}
\hline & \multicolumn{1}{c}{ Alto } & \multicolumn{1}{c}{ Medio } & Bajo \\
Cansancio emocional & $1(7 \%)$ & $6(43 \%)$ & $7(50 \%)$ \\
Realización personal & $1(7 \%)$ & $6(43 \%)$ & $7(50 \%)$ \\
Despersonalización & $3(21,5 \%)$ & $3(21,5 \%)$ & $8(57 \%)$ \\
Total & 5 & 15 & 22 \\
\hline
\end{tabular}

Tabla 10. Número de casados y porcentaje según niveles de puntuación (n: 12)

\begin{tabular}{|lllc|}
\hline & \multicolumn{1}{c}{ Alto } & Medio & Bajo \\
Cansancio emocional & $0(0 \%)$ & $6(29 \%)$ & $6(24 \%)$ \\
Realización personal & $0(0 \%)$ & $5(42 \%)$ & $7(58 \%)$ \\
Despersonalización & $2(17 \%)$ & $3(58 \%)$ & $7(58 \%)$ \\
Total & 2 & 14 & 20 \\
\hline
\end{tabular}

Tabla 12. Número de participantes que hacen turnos de urgencia y porcentaje según niveles de puntuación (n: 33)

\begin{tabular}{|lccc|}
\hline & Alto & Medio & \multicolumn{1}{c|}{ Bajo } \\
\hline Cansancio emocional & $19(58 \%)$ & $8(24 \%)$ & $6(18 \%)$ \\
Realización personal & $7(21 \%)$ & $14(42 \%)$ & $12(36 \%)$ \\
Despersonalización & $16(48 \%)$ & $8(24 \%)$ & $9(27 \%)$ \\
Total & 42 & 30 & 27 \\
\hline
\end{tabular}

una prevalencia de un 39\%, mientras que el CE mostró una prevalencia del 50\% (6); la baja RP, el 33\% (4), y DE el $34 \%$ (4) (Tabla 11)

Respecto a los que hacen turno de urgencia se observa una prevalencia de un 73\% mientras que el CE mostró una prevalencia del $82 \%$ (27); la baja RP, el 63\% (21), y la DE el $72 \%$ (24) (Tabla 12).

Respecto a los que duermen menos de $7 \mathrm{~h}$ se observa una prevalencia de un 68\% mientras que el CE mostró una prevalencia del 80\% (28); la baja RP el 57\% (20), y la DE el 66\% (23) (Tabla 13). Respecto a los que duermen más de 7 h se observa una prevalencia de un 54\%, mientras que el CE mostró una prevalencia del 50\% (4); la baja RP, el 62\% (5), y la DE el 50\% (4) (Tabla 14).

Tabla 7. Número de sujetos sin hijos y porcentaje según niveles de puntuación (n: 31)

\begin{tabular}{|lrrr|}
\hline & Alto & Medio & \multicolumn{1}{c}{ Bajo } \\
Cansancio emocional & $20(65 \%)$ & $7(22 \%)$ & $4(13 \%)$ \\
Realización personal & $5(16 \%)$ & $13(42 \%)$ & $13(42 \%)$ \\
Despersonalización & $15(48 \%)$ & $7(23 \%)$ & $9(29 \%)$ \\
Total & 40 & 27 & 26 \\
\hline
\end{tabular}

Tabla 9. Número de solteros y porcentaje según niveles de puntuación (n: 29)

\begin{tabular}{|lccr|}
\hline & Alto & Medio & \multicolumn{1}{c}{ Bajo } \\
Cansancio emocional & $20(69 \%)$ & $5(17 \%)$ & $4(14 \%)$ \\
Realización personal & $4(14 \%)$ & $13(45 \%)$ & $12(41 \%)$ \\
Despersonalización & $13(45 \%)$ & $7(24 \%)$ & $9(31 \%)$ \\
Total & 37 & 25 & 25 \\
\hline
\end{tabular}

Tabla 11. Número de participantes que no hacen turnos de urgencia y porcentaje según niveles de puntuación (n: 12)

\begin{tabular}{|lllc|}
\hline & Alto & Medio & Bajo \\
Cansancio emocional & $2(17 \%)$ & $4(33 \%)$ & $6(50 \%)$ \\
Realización personal & $1(8 \%)$ & $3(25 \%)$ & $8(67 \%)$ \\
Despersonalización & $2(17 \%)$ & $2(17 \%)$ & $8(66 \%)$ \\
Total & 5 & 9 & 22 \\
\hline
\end{tabular}

Tabla 13. Número de participantes que duermen menos de $7 \mathrm{~h}$ y porcentaje según niveles de puntuación (n: 35)

\begin{tabular}{|lccr|}
\hline & Alto & Medio & \multicolumn{1}{c|}{ Bajo } \\
\hline Cansancio emocional & $19(54 \%)$ & $9(26 \%)$ & $7(20 \%)$ \\
Realización personal & $7(20 \%)$ & $13(37 \%)$ & $15(43 \%)$ \\
Despersonalización & $16(46 \%)$ & $7(20 \%)$ & $12(34 \%)$ \\
Total & 42 & 29 & 34 \\
\hline
\end{tabular}




\section{ARTÍ́CULO ORIGINAL}

Tabla 14. Número de participantes que duermen más de 7 h y porcentaje según niveles de puntuación (n: 8)

\begin{tabular}{|llll|}
\hline & Alto & Medio & Bajo \\
Cansancio emocional & $1(12 \%)$ & $3(38 \%)$ & $4(50 \%)$ \\
Realización personal & $1(12 \%)$ & $4(50 \%)$ & $3(38 \%)$ \\
Despersonalización & $2(25 \%)$ & $2(25 \%)$ & $4(50 \%)$ \\
Total & 4 & 9 & 11 \\
\hline
\end{tabular}

Existen diferencias estadísticamente significativas en algunas dimensiones de Burnout según variables sociodemográficas. Se obtuvieron diferencias en CE y DE entre hombres y mujeres, solteros y casados, sujetos sin hijos y con hijos, no hace turnos de urgencia, obteniendo niveles más altos en las mujeres, en solteros, en sujetos sin hijos y los que hacen turnos de urgencia (Tabla 15$)$.

Tabla 15. Tabla comparativa de variables sociodemográficas según dimensión.

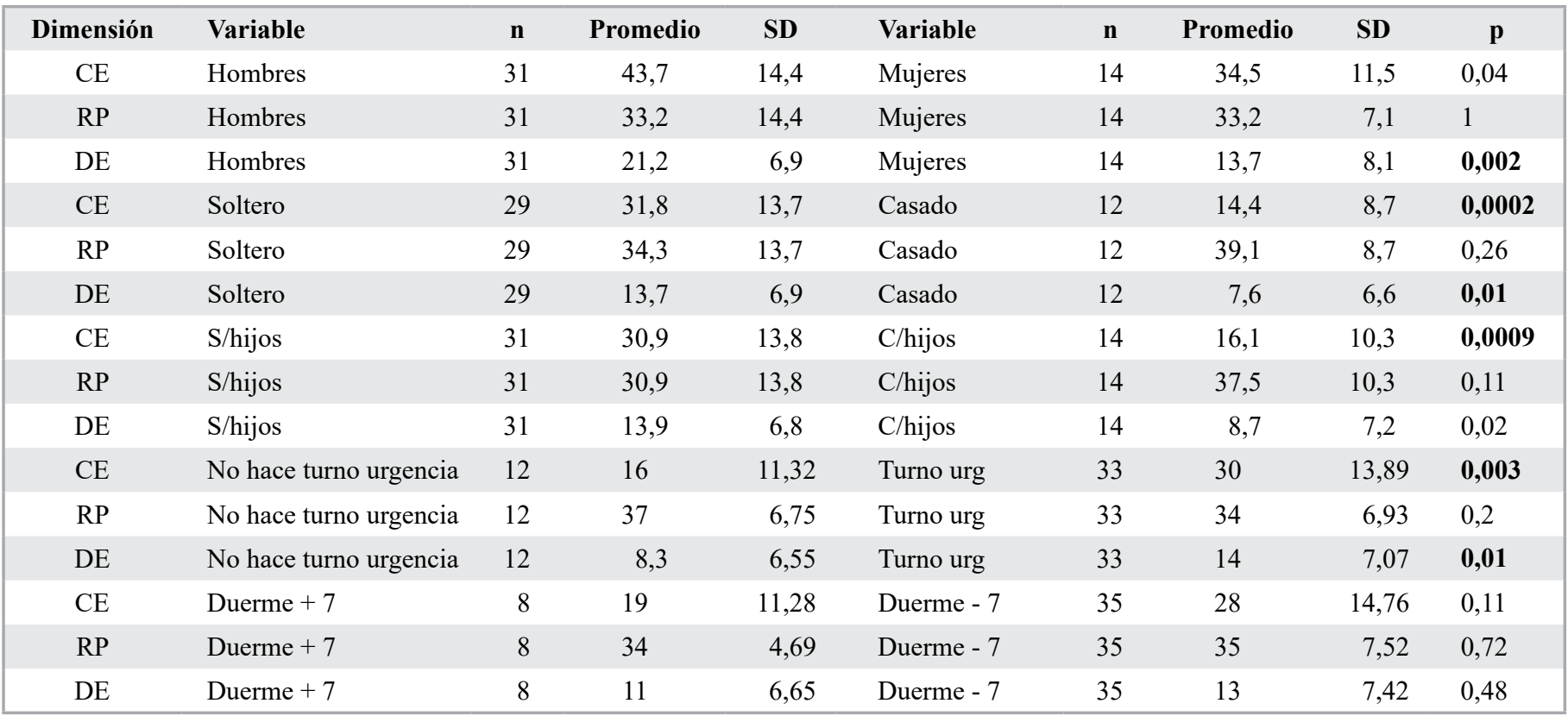

\section{Discusión}

Este es uno de los primeros reportes acerca de la prevalencia de síndrome de Burnout en un complejo de cirugía académico-asistencial en Chile. Aquí se plantean desafíos importantes, ya que es una alianza entre una universidad pública (Universidad de La Frontera) y un hospital de alta complejidad, público, autogestionado (Hospital Hernán Henríquez Aravena).

Los internos de cirugía y residentes de la Universidad de La Frontera son muy importantes en el funcionamiento asistencial del HHHA ya que dentro de su labor asistencial muchos consideran que sería difícil mantener el trabajo diario del HHHA sin su trabajo. Ellos trabajan bajo la supervisión tanto de docentes de la Universidad de La Frontera, como de cirujanos del HHHA. La relación de los internos y residentes de los hospitales en Chile es diferente a lo que sucede en países como Estados Unidos: no reciben salario por su actividad asistencial, no tienen beneficios de salud, ni previsionales y no tienen definida una carga horaria máxima a la semana.

Nuestros datos de consistencia interna son aceptables, en coherencia con los presentados en otras investigaciones ${ }^{30,31}$.

Los resultados respecto al cansancio emocional tienen sentido ya que este lleva a un distanciamiento emocional y cognitivo en las actividades diarias, con la consecuente incapacidad de responder a demandas y de dar espacio a la realización personal. La realización personal que se encuentra ya en niveles medios, es un riesgo de poder llegar a un agotamiento emocional y una consecuente despersonalización. Al no tenerla, dificulta la motivación con un efecto directo en la formación de los 
futuros médicos, ya que se reduce la tolerancia ante las dificultades que los estudiantes presenten en su formación.

En nuestra investigación la prevalencia global de síndrome de Burnout en un servicio de cirugía académico asistencial fue de $64,4 \%$; siendo mayor en los internos de medicina. Esto puede ser debido a que son el grupo con menor experiencia laboral, a que muchas veces la carga académica y asistencial es alta, y a la poca o nula preparación para manejo del estrés que actualmente contempla la mayoría de mallas curriculares de medicina en Chile.

Si comparamos los hallazgos de nuestro estudio con estudios similares, encontramos que un estudio realizado en Australia demostró una prevalencia de síndrome de Burnout en internos de 69\%, menor que la encontrada en el presente estudio (79\%). Los estudios realizados en Australia también muestran una prevalencia mayor en los internos cuando se comparan con otros grupos de trabajadores de la salud. Además, se encontró en el presente estudio que la prevalencia es mayor en aquellas rotaciones de mayor carga laboral y mayor nivel de responsabilidad como es el área de cirugía en nuestro hospital ${ }^{32}$.

Dentro de los aspectos que clásicamente se han asociado a la probabilidad de síndrome de Burnout están los trabajos en horas nocturnas; lo cual ha hecho que muchas universidades en Estados Unidos limiten las horas de trabajo nocturno de los estudiantes de medicina ${ }^{33}$. Sin embargo, las actividades nocturnas tienen un papel importante en el aprendizaje de la medicina y el tratamiento adecuado de situaciones complejas y emergencias a las que se verán expuestos los futuros médicos.

Un estudio que evaluó residentes de cirugía mostró una prevalencia de Burnout de 57,9\%, menor que la mostrada en nuestro estudio que fue de $63 \%$. Al igual que en nuestro estudio el tener hijos puede ser considerado un factor protector. Nuestro esquema de residencia en Chile plantea algunos factores que pudieran explicar esta mayor prevalencia: nuestros programas de residencia duran habitualmente sólo 3 años, lo cual plantea que nuestros residentes tienen un tiempo que pudiera considerase limitado para alcanzar las competencias para una vida laboral. Una cantidad importante de conocimientos y habilidades son adquiridos en los turnos nocturnos y todavía hay una modalidad de residencia "autofinanciada" que no recibe pago, ni ayuda para las cotizaciones de salud; esto plantea que el residente de esta modalidad debe gastar sus ahorros para poder mantenerse durante este período, y esto impone una mayor presión.

Otra de las particularidades de este escenario social hospitalario dice relación con que muchas veces el médico debe enfrentar el juicio social de "profesional médico deshumanizado" con una imagen negativa, sin embargo, esta percepción es frecuentemente desde una mirada del desconocimiento respecto al reflejo de una respuesta prolongada al estrés ya que estos profesionales enfrentan múltiples estresores entre los que destacan turnos rotatorios (que influye en los ritmos neurofisiológicos) ${ }^{35}$, sentimientos de infravaloración de su trabajo por los demás, escasa posibilidad de predecir o graduar las demandas asistenciales, la necesidad de tomar decisiones importantes en poco tiempo, falta de especificidad de funciones y tareas, lo que supone conflicto, problemas en las relaciones interpersonales, falta de autonomía y autoridad en el trabajo para poder tomar decisiones, rápidos cambios tecnológicos, situaciones límites que se relacionan directamente con el paciente, con su tratamiento, su muerte, entre muchos otros ${ }^{36-40}$.

En cuanto a la prevalencia de Burnout, dentro de los cirujanos fue del $48 \%$, lo cual parece ser un poco más baja que la mostrada en otros estudios ${ }^{41}$. Hay que tener en cuenta que este síndrome ha sido un predictor de la satisfacción con la profesión de los cirujanos ${ }^{41,42}$. Se han planteado opciones para la prevención y el tratamiento de este en el personal de salud. Dentro de las investigaciones existe una revisión sistemática del grupo Cochrane para encontrar factores que puedan prevenir el estrés en trabajadores de la salud. Este estudio clasifica las intervenciones en entrenamiento cognitivo-conductual (ECC), relajación física y mental, combinación de ECC y relajación; e intervenciones organizacionales. Cabe destacar que la calidad metodológica de la mayoría de los estudios es baja y que ECC y relajación física y mental muestra reducir el estrés al compararlo con el grupo de no intervención ${ }^{43}$.

En este grupo de sujetos existe la probabilidad de que se constituyan en protectores de Burnout el ser casados, tener hijos, que no realice turnos de urgencia, el ser hombre, lo que coincide con otras investigaciones en Latinoamérica ${ }^{44,45}$.

Es posible que estar casado y con hijos sea protector debido a la importancia de las relaciones interpersonales en el enfrentamiento del estrés y la prevención del síndrome. El no realizar turnos de urgencia posiblemente tenga relación con las horas de sueño; este factor ha sido asociado en otras investigaciones como factor protector46. En otros estudios el uso de estrategias de afrontamiento tiene un papel protector; aunque no totalmente del cansancio emocional, pero sí de la despersonalización y de la merma en la realización personal ${ }^{47}$.

Existe un estudio realizado por este equipo que 
va en búsqueda de factores presentes en el ambiente laboral que protegen y benefician la salud en situaciones de estrés percibidos por el personal de salud que trabaja en centros de atención hospitalaria pública y privada de la Región de la Araucanía, factores que podrían ser modificados por los administradores en salud. Se revelan 5 áreas que se relacionan con: 1) Organización: apoyar la capacitación de sus funcionarios con tiempo y flexibilidad horaria, proporcionar los recursos materiales y tecnológicos necesarios para la labor; 2) Puesto de trabajo: coherencia entre el rol profesional y las actividades que se realizan, escritas y definidas las funciones y objetivos de cada puesto de trabajo; 3) Jefatura: involucrarse en la labor de sus funcionarios, ser recta y justa; 4) Ambiente físico: ventanas que permitan mirar el exterior, espacios para desarrollar actividades como atención a la familia o alguna discusión grupal; 5) Trabajo en equipo y ambiente psicosocial: un lenguaje sin gritos ni estridencias dentro del equipo, un ambiente de trabajo entretenido y de buen humor, el respeto al trabajo de los otros ${ }^{48}$. Actualmente se trabaja en un modelo de medida que caracteriza a los ambientes hospitalarios protectores de estrés.

Dentro de las terapias para disminuir la prevalencia de Burnout existen algunas derivadas de la psicología y las religiones orientales, un poco alejadas de la realidad quirúrgica: la conciencia plena (mindfulness), la resiliencia y la gratitud. Además de reducir el Burnout, mejoran la calidad de vida de los profesionales que lo practican. Existen cursos específicos de entrenamiento en compasión, conciencia plena y resiliencia. El otro factor protector para el desarrollo de Burnout es el tiempo de sueño; aspecto difícil de regular en el entrenamiento y la práctica quirúrgica $^{49}$.

\section{Conclusión}

La prevalencia global de Síndrome de Burnout fue más alta que la encontrada en otras investigaciones internacionales; siendo los internos de medicina el grupo con mayor prevalencia.

Las direcciones académicas y hospitalarias deben sensibilizarse sobre la urgencia de integrar medidas de prevención, tratamiento ( $\mathrm{si}$ el caso lo requiere) y apoyo hacia a los médicos.

El problema del Síndrome de Burnout en este servicio quirúrgico de Chile es real; según el modelo docente asistencial de las universidades públicas en Chile, afecta a todos los actores en el modelo de atención de salud: internos, residentes y staff. Ade- más, está asociado con la satisfacción del paciente, la calidad del cuidado y los errores de la medicina, esto hace que las estrategias para enfrentarlo y prevenirlo sean prioridad en las organizaciones médicas.

Respecto al trabajo en turnos, se plantea la paradoja de cómo diseñar estrategias educacionales que permitan que los internos y residentes adquieran estas importantes competencias, limitando la presión para disminuir las tasas de Burnout. Bajo estas condiciones no hay capacidad de recuperarse física ni mentalmente para enfrentar las exigencias laborales hospitalarias.

Se acepta que el factor central desencadenante es el excesivo agotamiento emocional que gradualmente lleva a un estado de distanciamiento emocional y cognitivo en sus actividades diarias, con la consecuente incapacidad de responder a las demandas de servicio. En este distanciamiento ocurre una despersonalización, indiferencia y actitudes vinculadas al sarcasmo y la ironía hacia las responsabilidades o hacia las personas, una tendencia de no creer en la sinceridad del ser humano. Por tanto, se deben tomar medidas para intentar reducir la prevalencia de éste síndrome en nuestros internos y residentes principalmente. Hemos planteado intervenciones con cursos y prácticas de meditación consciente y esperamos reportar los resultados en los siguientes años.

\section{Responsabilidades éticas}

Protección de personas y animales. Los autores declaran que para esta investigación no se han realizado experimentos en seres humanos ni en animales.

Confidencialidad de los datos. Los autores declaran que en este artículo no aparecen datos de pacientes.

Derecho a la privacidad y consentimiento informado. Los autores declaran que en este artículo no aparecen datos de pacientes.

\section{Financiación}

Los autores declaran no haber recibido ninguna financiación para la realización de este trabajo.

\section{Conflicto de intereses}

Los autores declaran no tener ningún conflicto de intereses. 


\section{Bibliografía}

1. Campbell DA Jr, Sonnad SS, Eckhauser FE, Campbell KK, Greenfield LJ. Burnout among American surgeons. Surgery 2001;130: 696-702.

2. Spickard A Jr, Gabbe SG, Christensen JE. Mid-career burnout in generalist and specialist physicians. JAMA 2002;288:1447-50.

3. Dyrbye LN, Thomas MR, Huschka MM, Lawson KL, Novotny PJ, Sloan JA, et al. A multicenter study of burnout, depression, and quality of life in minority and nonminority US medical students. Mayo Clin Proc. 2006;81:1435-42.

4. Thomas NK. Resident burnout. JAMA 2004;292:2880-9.

5. McCray LW, Cronholm PF, Bogner HR, Gallo JJ, Neill RA. Resident physician burnout: is there hope? Fam Med. 2008;40:626-32.

6. West CP, Shanafelt TD, Kolars JC. Quality of life, burnout, educational debt, and medical knowledge among internal medicine residents. Jama 2011;306:95260.

7. Businger A, Stefenelli U, Guller U. Prevalence of burnout among surgical residents and surgeons in Switzerland Archives of Surgery 2010;145:1013-6.

8. García M. Evaluación del burnout: estudio de la fiabilidad, estructura empírica y validez de la escala EPB. Ansiedad y Estrés 1995;1:219-29.

9. Maslach C, Schaufeli WB, Leiter MP. Job burnout. Annu Rev Psychol. 2001;52:397422.

10. Austria-Corrales F, Cruz-Valdés B, Herrera-Kiengelher L, Vázquez-García JC, Salas-Hernández J. [Burnout syndrome among medical residents during the influenza A H1N1 sanitary contigency in Mexico]. Gaceta medica de México 2011;147:97-103.

11. Dyrbye LN, West CP, Satele D. Burnout among U.S. medical students, residents, and early career physicians relative to the general U.S. population. Academic medicine: journal of the Association of American Medical Colleges 2014;89:44351.

12. Shanafelt TD, West CP, Sloan JA, Novotny PJ, Poland GA, Menaker R, et al. Career fit and burnout among academic faculty. Arch Intern Med. 2009;169:9905. 11. Crane M. Why burned-out doctors get sued more often. Med Econ 1998; 75 : 215-8.

13. Shanafelt TD, Balch CM, Bechamps G, Russell T, Dyrbye L, Satele D, et al. Burnout and medical errors among American surgeons. Ann Surg. 2010;251:995-1000.

14. Weinstein L, Wolfe HM. The downward spiral of physician satisfaction: an attempt to avert a crisis within the medical profession. Obstet Gynecol 2007;109:1181-3

15. Fahrenkopf AM, Sectish TC, Barger LK, Sharek PJ, Lewin D, Chiang VW, et al. Rates of medication errors among depressed and burnt out residents: prospective cohort study BMJ 2008;336:488-91.

16. West CP, Tan AD, Habermann TM, Sloan JA, Shanafelt TD. Association of resident fatigue and distress with perceived medical errors. JAMA 2009;302:1294300 .

17. Shanafelt TD, Balch CM, Bechamps G, Russell T, Dyrbye L, Satele D, et al. Burnout and medical errors among American surgeons. Ann Surg. 2010;251:995-1000.

18. Williams B. Pursuing their passions. When they're not seeing patients, these doctors are living out their non-medical dreams. Tenn Med. 2007;100:28-35.

19. Foster C. Why doctors should get a life. J R Soc Med. 2009;102:519-20.

20. Pedrals N, Rigotti A, Bitran M. Aplicando psicología positiva en educación médica. Rev Med Chile 2011;139:941-9.

21. Peiró JM. El estrés laboral: una perspectiva individual y colectiva, Catedrático de psicología social y las organizaciones. [en línea] 2001, 13; 18 38 [Fecha de consulta: 26 de enero de 2014]. Disponible en: http://www.insht. es/InshtWeb/Contenidos/Documentacion/ TextosOnline/Rev_INSHT/2001/13/ seccionTecTextCompl2.pdf).

22. Programa de naciones unidas para el desarrollo, UFRO, Gobierno de Chile. Índice de desarrollo humano. Araucanía. 2003, número 8

23. Albaladejo R, Villanueva R, Ortega $P$, Astasio P, Calle ME, Domínguez V. Síndrome de Burnout en el personal de enfermería de un hospital de Madrid. Rev Esp Salud Pública 2004;78:505-6.

24. Bakker A, Killmer CH, Siegrist J, Schaufeli WB. Effort-reward imbalance and burnout among nurses Journal of Advanced Nursing 2008;4:884-91. Article first published online: $9 \mathrm{de}$ octubre de 2008 DOI: $10.1046 /$ j.13652648.2000.01361.x

25. Maslach C, Jackson SE. The measurement of experienced burnout. Journal of
Occupational Behaviour 1981;2:99-113. doi: 10.1002/job.4030020205 Maslach C, Jackson SE (1996). The Maslach Burnout Inventory. $3^{\text {a }}$ ed. Palo Alto, CA: Consulting Psychologist Press.

26. Maslach C, Schaufeli W, Leiter MP. Job burnout. Annual Review of Psychology 2001;52:397-422. doi: 10.1146/annurev. psych.52.1.397.

27. Cañadas-de la Fuente GA, San Luis C, Lozano LM, Vargas C, García I, de la Fuente EI. Evidencia de validez factorial del Maslach Burnout Inventoryy estudio de los niveles de burnout en profesionales sanitarios. Rev Lat Psic 2014;46:44-52.

28. George D, Mallery M. Using SPSS for windows step by step: A simple guide and reference. Boston, MA: Allyn y Bacon. 2003; p. 231.

29. Aranda-Beltrán C, López-Gónzalez JL, Barraza-Salas JH. Factores psicosociales y síndrome de burnout en trabajadores de la industria de la transformación de la masa, Tepic, México. Rev Colomb Psiquiat. 2013;42:167-72.

30. West CP, Dyrbye LN, Sloan JA, Shanafelt TD. Single item measures of emotional exhaustion and depersonalization are useful for assessing burnout in medical professionals. J Gen Intern Med. 2009;24:1318-21. doi: 10.1007/s11606009-1129-z. Epub 2009 Oct 3

31. McAbee JH, Ragel BT, McCartney S, Jones GM, Michael LM 2nd, DeCuypere $\mathrm{M}$, et al. Factors associated with career satisfaction and burnout among US neurosurgeons: results of a nationwide survey. J Neurosurg. 2015;123:161-73. doi: 10.3171/2014.12.JNS141348.

32. Parr JM, Pinto N, Hanson M, Meehan A Moore PT. Medical Graduates, Tertiary Hospitals, and Burnout: A Longitudinal Cohort Study. Ochsner J. 2016;16:22-6.

33. Karani R. Enhancing the Medical School Learning Environment: A Complex Challenge. J Gen Intern Med 2015;30:1235-6. doi: 10.1007/s11606015-3422-3.

34. Malik AA, Bhatti S, Shafiq A, Khan RS, Butt UI, Bilal SM, et al. Burnout among surgical residents in a lower-middle income country-Are we any different? Ann Med Surg. (Lond). 2016;9:28-32. doi: 10.1016/j.amsu.2016.05.012. eCollection 2016.

35. Ganster DC, Fox ML, Dwyer DJ Explaining employees'health care costs: a prospective examination of stressful job demands, personal control, and physiological reactivity. J Appl Psychol. 2001;86:954-64. 


\section{ARTÍ́CULO ORIGINAL}

36. de Quirós-Aragón B, Labrador-Encinas M. Evaluación del estrés laboral y burnout en los servicios de urgencia. International Journal of Clinical and Health Psychology 2007;7:323-35, Asociación Española de Psicología Conductual. España. Disponible en: http//www.redalyc.org/ artículo.oa?id=33717060005.

37. Gray-Toft P, Anderson JG. Stress among hospital nursing staff: its causes and effects. Soc Sci Med. 1981;15:639-47.

38. Hipwell AE, Tyler PA. Sources of stress and dissatisfaction among nurses in four hospital environments. Br J Med Psychol. 1989;62:71-9.

39. Harris P. The nurses stress index. Work Stress 1989;3:335-46.

40. Quinn MA, Wilcox A, Orav EJ, Bates DW, Simon SR. The relationship between perceived practice quality and quality improvement activities and physician practice dissatisfaction, professional isolation, and work-life stress. Med Care 2009;47:924-8.

41. Helewa RM, Kholdebarin R, Hochman
DJ. Attending surgeon burnout and satisfaction with the establishment of a regional acute care surgical service. Can J Surg. 2012;55:312-6.

42. Shanafelt TD, Balch CM, Bechamps GJ, Russell T, Dyrbye L, Satele D, et al. Burnout and career satisfaction among American surgeons. Ann Surg. 2009;250:463-71. doi: 10.1097/ SLA.0b013e3181ac4dfd.

43. Ruotsalainen JH, Verbeek JH, Mariné A, Serra C. Preventing occupational stress in healthcare workers. Cochrane Database Syst Rev. 2014 Dec 8;(12):CD002892. doi: 10.1002/14651858.CD002892.pub4. Review. Update in: Cochrane Database Syst Rev. 2015;(4):CD002892.

44. Ticas AR, Medina M, Mesa X, Paredes Y, Barahona Y, Sierra M. Estudio de síndrome de "burnout", depresión y factores asociados en los practicantes internos del hospital escuela. Rev Fac Cienc Med. 2012;9:14-20.

45. Galván ME, Vassallo JC, Rodríguez SP, Otero P, Montonati MM, Cardigni G, et al. Síndrome de desgaste profesional (burnout) en médicos de unidades de cuidados intensivos pediátricos en la Argentina. Revista de la Sociedad Boliviana de Pediatría 2014;53:29-36.

46. Wisetborisut A, Angkurawaranon C, Jiraporncharoen W, Uaphanthasath R, Wiwatanadate P. Shift work and burnout among health care workers. Occupational Medicine 2014;64:279-86.

47. Valdés CB, Austria-Corrales F, HerreraKiengelher L, Carlos J. Estrategias activas de afrontamiento: un factor protector ante el síndrome de burnout "o de desgaste profesional" en trabajadores de la salud. Neumol Cir Torax 2010;69:137-42.

48. Astudillo Díaz P, Alarcón Muñoz AM. "Protectores de estrés laboral: percepción del personal de enfermería y médicos, Temuco, Chile." Ciencia y enfermería 2009;15:111-22.

49. Dissanaike $\mathrm{S}$. How to prevent burnout (maybe). Am J Surg. 2016;212:1251-5. doi: 10.1016/j.amjsurg.2016.08.022. Epub 2016 Oct 20 . 